\title{
Delayed Cerebral Ischemia in Aneurysmal Subarachnoid Hemorrhage: Proposal of an Evidence-Based Combined Clinical and Imaging Reference Standard
}

\author{
P.C. Sanelli, S. Kishore, A. Gupta, H. Mangat, A. Rosengart, H. Kamel, and A. Segal
}

\begin{abstract}
SUMMARY: Aneurysmal subarachnoid hemorrhage is associated with high morbidity and mortality, with delayed neurologic deficits from delayed cerebral ischemia contributing to a large portion of the adverse outcomes in this patient population. There is currently no consensus reference standard for establishing the diagnosis of delayed cerebral ischemia either in the research or clinical settings, ultimately limiting strategies for preventing delayed infarction and permanent neurologic deficits. There are currently both clinical and imaging-based criteria for the diagnosis of delayed neurologic deficits and vasospasm, respectively, however, neither clinical nor angiographic assessment alone has been shown to identify patients who develop adverse outcomes from delayed infarction. Thus, the purpose of this work is to propose a 3-tiered combined imaging and clinical reference standard based on evidence from the literature to standardize the diagnosis of delayed cerebral ischemia, both to allow consistency across research studies and to ultimately improve outcomes in the clinical setting.
\end{abstract}

ABBREVIATIONS: aSAH = aneurysmal subarachnoid hemorrhage; $\mathrm{DCI}=$ delayed cerebral ischemia; $\mathrm{MRP}=\mathrm{MR}$ perfusion

$\mathrm{A}^{\mathrm{n}}$ neurysmal subarachnoid hemorrhage (aSAH) is associated with high morbidity and mortality. ${ }^{1,2}$ The first 2 weeks following aSAH are critical in the management of these patients because they are prone to develop several life-threatening complications, including delayed neurologic deficits, ${ }^{3}$ which often arise from delayed cerebral ischemia (DCI), a major contributor to the adverse outcomes in this population. ${ }^{3-5}$ Delayed cerebral ischemia manifests in approximately $30 \%$ of patients with aSAH and typically occurs between days 4 and 9 after the initial hemorrhage, though it can range from 3 to 14 days.

There remains a lack of standard criteria for defining DCI in the clinical setting, ${ }^{3,6,7}$ with a recent literature review describing at

From the Departments of Radiology, Division of Neuroradiology (P.C.S., S.K., A.G.); Public Health (P.C.S.); and Neurology, Division of Stroke and Critical Care (H.M.,

A.R., H.K., A.S.), NewYork-Presbyterian Hospital-Weill Cornell Campus, New York, New York.

P. Sanelli and S. Kishore are co-first authors.

This work was supported by grant 5K23NS058387 from the National Institute of Neurological Disorders and Stroke, a component of the National Institutes of Health.

The views herein are solely the responsibility of the authors and do not necessarily represent the official view of the National Institute of Neurological Disorders and Stroke or the National Institutes of Health.

Please address correspondence to Pina C. Sanelli, MD, MPH, Department of Radiology, Weill Cornell Medical College, NewYork-Presbyterian Hospital, 525 East 68th St, Starr Pavilion, Box 141, New York, NY 10065; e-mail: pcs9001@med.cornell.edu

- Indicates open access to non-subscribers at www.ajnr.org

http://dx.doi.org/10.3174/ajnr.A3782 least 8 terms to define the concept of DCI in aSAH. ${ }^{6}$ Debate over the role of clinical and imaging assessments in defining DCI has occurred for both clinical and research purposes. ${ }^{3,6,8-10}$ For example, although the terms "DCI" and "vasospasm" have been used interchangeably, attempts have been made to distinguish DCI from vasospasm, with the former often determined clinically, and the latter, radiographically, ${ }^{6}$ because not all patients with clinical neurologic deficits have angiographic vasospasm and not all patients with angiographic vasospasm have neurologic deficits that correspond to the arterial territory of vasospasm. ${ }^{11,12}$ Additionally, while severe vasospasm may cause decreased cerebral perfusion, a substantial percentage of patients develop infarction without evidence of vasospasm, suggesting that DCI should be defined as a pathologic process, of which vasospasm may represent a contributing factor. ${ }^{12,13}$

Thus, the aim of this article is to propose an evidence-based reference standard for DCI that incorporates both clinical assessments of neurologic deterioration and imaging assessments of vasospasm, perfusion deficits, and infarction to provide a consistent, uniform standard across a wide range of clinical and research applications. The classification of levels of evidence supporting this reference standard is based on the Levels of Evidence criteria proposed by the Oxford Centre of Evidence Based Medicine (www.cebm.net). ${ }^{14}$ Two independent reviewers assessed levels of evidence for each tier, and in the case of discordance, evidence level assignments were made by consensus. 


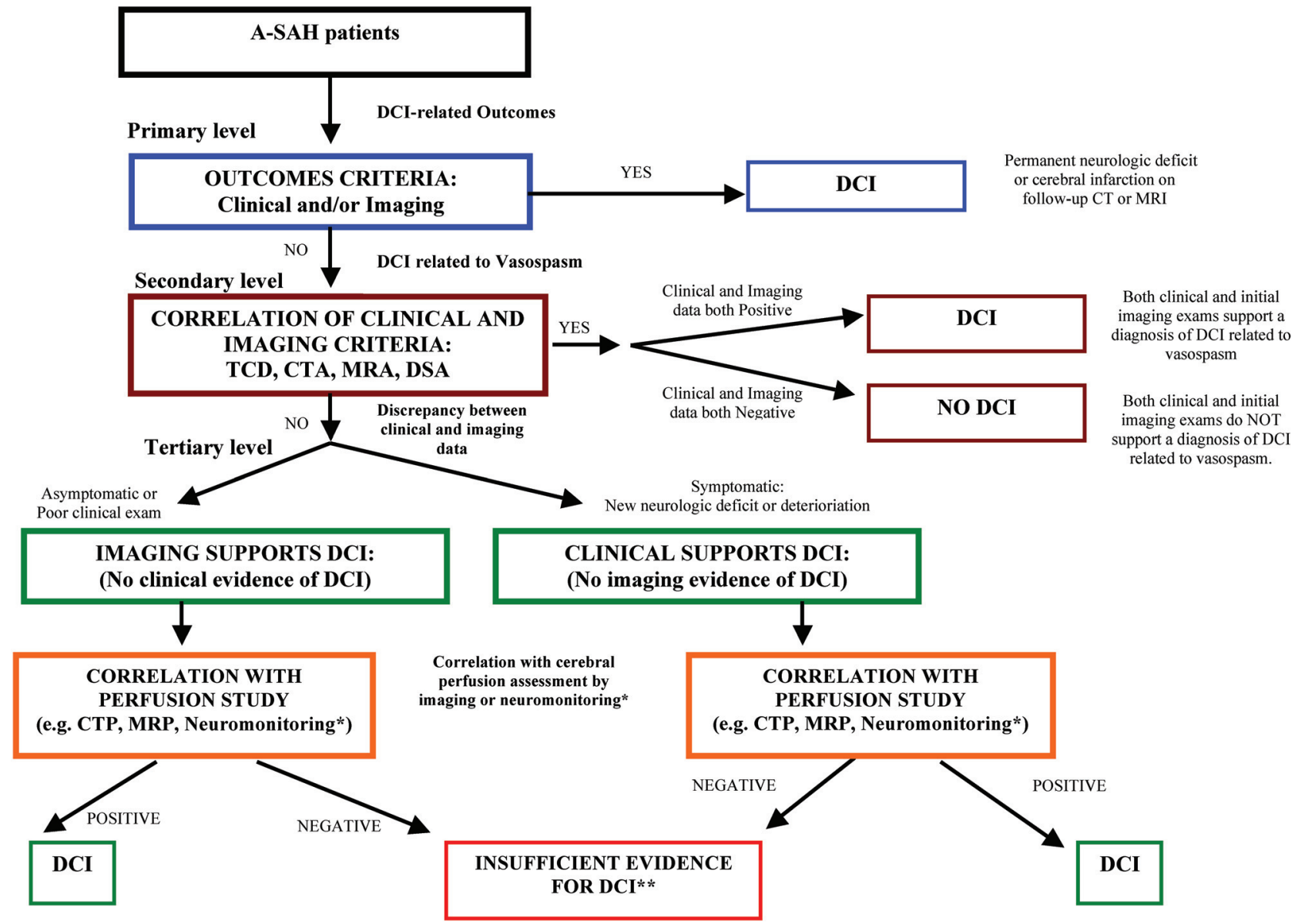

FIG 1. Proposed multitiered reference standard in DCI. Three-tiered DCl reference standard algorithm, ordered from top to bottom. Asterisk indicates neuromonitoring devices such as cerebral microdialysis and oximetry. Double asterisks indicate whether the reference standard is used for clinical assessment and treatment decisions based on the risk/benefit ratio for treatment. If there is low risk, treatment for DCl is recommended. If there is high risk, the patient should re-enter the algorithm.

\section{DESCRIPTION OF THE COMBINED CLINICAL AND IMAGING REFERENCE STANDARD}

(Algorithm displayed in Fig 1)

\section{Primary Level: Outcome-Based Criteria}

Summary. The primary level classifies patients as having DCI if a new infarction on imaging or new permanent neurologic deficit develops. A new infarction on imaging is determined on CT or MR imaging within 6 weeks after aSAH ictus that was not present on imaging up to 48 hours after aneurysm occlusion and was not attributable to other causes such as surgical clipping, endovascular treatment, ventricular catheter placement, intraparenchymal hematoma, or cerebral herniation. A new permanent neurologic deficit is determined on clinical examination as a new neurologic deficit distinct from the baseline examination performed immediately after aneurysm rupture or aneurysm occlusion and not attributable to other causes. Baseline neurologic examination must be considered after full cardiorespiratory, hemodynamic, and metabolic resuscitation as well as treatment of other factors such as seizures and hydrocephalus. Patients who do not meet either criterion are referred to the secondary level, as described in a subsequent section.

Evidence: Level 1A evidence exists to support these proposed outcomes-based criteria for determining DCI.

An ideal reference standard should reliably identify patients with a high risk of poor outcomes who may benefit from intervention. In large prospective cohort studies, the greatest predictors of severe disability or death at 3 months were a new focal neurologic deficit, a new infarction on follow-up imaging, or both. ${ }^{6,15,16}$ Additionally, a large systematic review and meta-analysis of all randomized placebo-controlled trials evaluating the efficacy of protective strategies in aSAH concluded that a reduced incidence of cerebral infarction is significantly associated with improved functional outcome. ${ }^{17}$ In fact, new cerebral infarction alone was as strongly correlated with poor 3-month functional outcome as the combination of a new neurologic deficit and corresponding ischemic changes on follow-up neuroimaging. ${ }^{6}$ Furthermore, cerebral infarction on noncontrast CT was the primary outcome measure in the early trials of nimodipine, an agent with strong evidence for neuroprotection of DCI. ${ }^{18}$ 
While angiographic vasospasm has traditionally been the primary focus of interventions and prediction of outcomes, the lack of evidence demonstrating improved outcomes with vasospasm prevention ${ }^{13,19}$ has led to incorporating this criterion combined with clinical correlation in the secondary level below.

\section{Secondary Level: Correlation of Clinical and Vascular Imaging Criteria}

Summary. The secondary level classifies patients as having DCI if both clinical deterioration and angiographic vasospasm occur. Clinical deterioration is determined by bedside examination and comprises the development of a new neurologic deficit (such as hemiparesis, hemiplegia, aphasia, depressed consciousness, and so forth), a decrease of at least 2 points on the Glasgow Coma Scale, or a decrease of at least 1 point in the motor score, lasting $>1$ hour at any point after aneurysm occlusion and not attributable to other causes. Vascular imaging for the evaluation of vasospasm includes imaging modalities, such as transcranial Doppler sonography, CTA, MRA, and DSA. Patients with neurologic deterioration and 1 imaging test supporting a diagnosis of vasospasm are classified as having DCI. On the other hand, patients without neurologic deterioration and 1 imaging test without findings of vasospasm are classified as not having DCI. However, patients with either positive clinical or imaging findings that do not correlate with each other are referred to the tertiary level, as described in a subsequent section.

Evidence: Level 1B evidence exists to support using clinical and vascular imaging data for determining DCI.

Evaluation of patients for DCI at the secondary level is most valuable in the clinical setting at the point of care when treatment decisions are made. The primary goal of treatment is to prevent cerebral infarction and permanent neurologic deficits. Thus, traditionally, imaging assessment of vasospasm has been used as a surrogate marker to assist in the diagnosis of DCI, especially given that neurologic deterioration is poorly evaluated in sedated or obtunded patients. Angiographic vasospasm, seen on DSA or CTA, is perhaps the most commonly used surrogate imaging marker in this patient population. Vasospasm has been shown to be strongly associated with DCI, cerebral infarction, poor outcome, and increased mortality within several retrospective and prospective cohort studies, including a post hoc analysis of data from the CONSCIOUS (Clazosentan to Overcome Neurological Ischemia and Infarct Occurring after Subarachnoid Hemorrhage)-1 trial. ${ }^{1,20-23}$ However, an analysis of data from 2 systematic reviews and a post hoc analysis did not demonstrate an improvement in outcome with a reduction in angiographic vasospasm. ${ }^{19}$ Evidence from both prospective and retrospective cohort studies suggests that patients with angiographic vasospasm and correlated symptoms have worse hospital complications and subsequent disability compared with angiographic vasospasm alone. ${ }^{9,16}$ However, there is less evidence to demonstrate the prognostic importance of angiographic vasospasm correlated with symptoms, thus placing this criterion at the secondary level. Although relatively inferior in terms of sensitivity and specificity, transcranial Doppler sonography evaluations of the intracranial vessels can also be performed at bedside to identify arterial nar- rowing in patients who may be too unstable for more advanced angiographic techniques such as CTA, MRA, or DSA. ${ }^{24,25}$

\section{Tertiary Level: Correlation of Physiologic Data with Clinical or Imaging Criteria}

Summary. The tertiary level classifies patients as having DCI if physiologic data correlates with either clinical deterioration or vasospasm. Patients with either clinical deterioration or vasospasm alone may undergo additional physiologic assessment of cerebral hemodynamics, either in the form of imaging such as CTP and MR perfusion (MRP) or neuromonitoring devices such as cerebral blood flow, oxygen tension monitoring, and cerebral microdialysis. Patients with findings suggestive of regional cerebral hypoperfusion or hypoxia that correlate with either clinical deterioration or vasospasm are classified as having DCI. Patients with clinical deterioration or vasospasm but normal physiologic data do not have sufficient evidence to be classified as having DCI.

Evidence: Levels of evidence to support using physiologic data for determining DCI range from $2 \mathrm{~A}$ to $3 \mathrm{~B}$, depending on the technique.

While there is at least moderate evidence supporting the importance of symptomatic vasospasm in DCI at the secondary level, the importance of isolated image-based diagnoses of vasospasm in the absence of clinical findings is somewhat controversial, especially in the absence of infarction. However, a subset of patients with asymptomatic vasospasm will develop asymptomatic ischemia and subsequent infarction. A large prospective cohort identified asymptomatic infarction in approximately $20 \%$ of patients with aSAH, and furthermore, these patients had a higher frequency of death and moderate-to-severe disability at 3 months relative to patients with symptomatic infarction. ${ }^{26}$ Thus, there may be a subset of patients with apparently asymptomatic vasospasm who are at high risk of eventually developing clinical evidence of DCI, especially those who are comatose or have a ventriculostomy catheter, small-volume aSAH, or ischemia in noneloquent brain ${ }^{26,27}$-all representing complicating factors that are not infrequently encountered in the intensive care setting. Identifying this high-risk subset of patients with asymptomatic vasospasm may prompt measures to implement therapies to prevent the eventual development of DCI.

Conversely, the identification of patients with DCI and clinical deterioration in the absence of vasospasm poses a different important diagnostic challenge. While neurologic deterioration is likely multifactorial in these patients, a subset will go on to develop infarction without vasospasm. A retrospective study of infarction patterns in patients with aSAH found that approximately $17 \%$ of patients developed infarcts without imaging evidence of vasospasm, and even in patients with imaging positive for vasospasm, infarcts also developed in areas away from the vasospastic territories. ${ }^{28}$ Thus, this level in the algorithm would attempt to identify ischemia in patients with asymptomatic vasospasm or neurologic deterioration without evidence of large-vessel vasospasm.

Perfusion imaging such as CTP and MRP or less common modalities such as xenon-CT provide physiologic imaging assessments of cerebral hypoperfusion and ischemia that could identify patients at risk for infarction. In a retrospective cohort of 96 pa- 
tients with aSAH, new CTP deficits seen as prolonged MTT and reduced $\mathrm{CBF}$ were significantly associated with subsequent infarction and permanent neurologic deficits. ${ }^{29}$ A smaller prospective study evaluating the test characteristics of CTP, CTA, and noncontrast CT obtained at baseline and after the onset of clinical deterioration determined that CTP had the best test performance for the subsequent diagnosis of DCI at discharge. ${ }^{30}$ Subsequently, systematic reviews evaluating CTP in aSAH within the broader context of diagnosing vasospasm and DCI found that relative CBF and MTT values correlated highly with subsequent DCI. ${ }^{25,31}$ Thus, there is level 2A evidence to support the role of CTP in the diagnosis of DCI.

Evidence to support the use of other imaging modalities to evaluate DCI is more limited. There are limited data evaluating the role of MRP in DCI; however, several small prospective cohort studies demonstrated that CTP, particularly CBF, correlates with MRP-derived values in the same patients within a close time interval, suggesting that MR imaging could also be used in this setting in case CTP is not performed. ${ }^{32,33}$ The data for the use of xenon-CT in DCI are even more limited; however, a small prospective cohort study in patients with poor-grade aSAH found that $\mathrm{CBF}$ reduction on xenon-CT was only moderately predictive of infarction in these patients and that not all reductions in $\mathrm{CBF}$ by this technique resulted in infarction. ${ }^{34}$ Thus, there is at best level 2B and 3B evidence for MRP and xenon-CT, respectively, for the diagnosis of ischemia in patients with aSAH. However, these imaging modalities are challenging to perform in this patient population due to scanner accessibility and patient contraindications.

Not all patients with aSAH undergo imaging to assess ischemia, particularly those who are unstable or have poor-grade conditions. Thus, noninvasive and invasive bedside monitoring devices such as cerebral microdialysis, brain tissue oxygenation monitoring (eg, the Licox system, Integra LifeSciences, Plainsboro, New Jersey), and other similar devices have been used to stratify patients at risk of ischemia. A systemic review evaluating the use of microdialysis in the assessment of cerebral ischemia in patients with aSAH found that while the use of the technology is increasing, there is substantial study heterogeneity, thereby limiting the evidence to support its utility. ${ }^{35}$ Nonetheless, a small prospective cohort of 44 patients found that a 2 -fold increase in ischemia-related metabolites from baseline at the time of acute neurologic deterioration was significantly associated with subsequent infarction and permanent neurologic deficits. ${ }^{36}$ Data for cerebral tissue oxygen monitoring are more limited, particularly in patients with aSAH. Several small prospective cohort studies demonstrated the potential utility of detecting hypoxia in aSAH by using tissue oxygenation monitoring. ${ }^{37-39}$ Thus, there is level $3 \mathrm{~A}$ evidence in support of cerebral microdialysis and level $3 \mathrm{~B} \mathrm{ev-}$ idence to support cerebral oxygen monitoring in patients with aSAH.

\section{Strengths and Limitations of Each Level}

Primary Level. The main strength of the primary level is its strong evidence using outcome-based criteria supported by systematic reviews and large observational cohort studies..$^{3,15,17,19}$ Thus, the primary level captures patients with the highest mortality and morbidity associated with DCI. Most important, this level em- phasizes specificity over sensitivity to accurately identify patients with DCI for treatment decisions. Additionally, patients with DCI who do not develop infarction or neurologic deficits cannot be misclassified at this level because these patients advance to the secondary level for further evaluation. Another strength at this level is the reproducibility in assessing patients with these welldefined outcome measures that are less prone to interobserver variability.

The main limitation at the primary level is the reduced applicability in guiding treatment decisions. In clinical practice, the goal of managing patients with aSAH is to avoid these devastating outcomes of infarction and functional disability. At this level, patients are classified as having DCI according to these criteria, thus limiting improvement in patient outcomes with treatment.

Secondary Level. The main strength of the secondary level is the combination of new neurologic deficits with imaging findings suggestive of angiographic vasospasm that have been shown to correlate with functionally relevant outcomes. ${ }^{9}$ Because these criteria can, in some cases, be evaluated before development of infarction and functional disability (ie, at a stage in which impending DCI is still preventable), classification of patients with DCI at this level should theoretically provide maximal benefit from treatment. The combination of both new neurologic deficits and evidence of angiographic vasospasm improves the specificity for identifying patients with DCI, given that neurologic assessment in patients with aSAH can be challenging and angiographic vasospasm does not necessarily correlate with DCI.

A limitation of the secondary level is that patients without new neurologic deficits and without angiographic vasospasm can be misclassified as having no DCI. Comatose or heavily sedated patients have limited clinical assessment and may have suboptimal imaging, resulting in false-negatives for DCI. Although the agreement of clinical and imaging findings improves the specificity for identifying patients with DCI for treatment, the sensitivity may not be optimized at this level for a subset of patients. The probability of correlation is dependent on the quality of each respective evaluation, and both the clinical and imaging assessments at this level are subject to interobserver variability. ${ }^{10,40,41}$

Tertiary Level. The main strength of the tertiary level is improving the sensitivity of the DCI diagnosis by further evaluating discordant clinical and imaging findings from the secondary level, such as in patients with asymptomatic vasospasm or neurologic decline without angiographic vasospasm. Most important, this level allows further evaluation of comatose patients with suboptimal clinical assessments who have angiographic vasospasm as well as symptomatic patients who have suboptimal imaging. These patients often have worse outcomes in comparison with patients with symptomatic DCI, possibly related to delayed treatment. ${ }^{26}$ At this level, all patients undergo physiologic assessment of cerebral perfusion and hypoxia to correlate with either clinical or imaging findings suggesting DCI. Thus, this level will include patients who may have been excluded from the diagnosis due to lack of sufficient evidence at the other 2 levels.

A potential limitation of the tertiary level is that the breadth of modalities used to assess ischemia-ranging from noninvasive imaging to invasive tissue monitoring — has variable strength of 
evidence to support their use. From an imaging standpoint, CTP has the strongest evidence to support its use in diagnosing DCI; clinically, cerebral microdialysis has some evidence to support its use despite inconclusive results from a systematic review of the literature. There is limited evidence to support the use of the remaining modalities in diagnosing DCI in patients with aSAH.

\section{Future Directions}

While there is no perfect reference standard for this complex disease process, this multitiered algorithm attempts to capture the complexity of clinical and imaging findings in DCI according to evidence-based criteria. Specificity is emphasized in this multitiered reference standard with respect to evidence-based clinically relevant outcomes at the primary level, which are particularly valuable in the research setting to potentially improve translation of research findings into clinical practice. Most important, this reference standard approach also incorporates levels of evidence with greater sensitivity for use in clinical settings. The model is heavily weighted toward criteria with supportive statistical evidence and, through a multitiered algorithm, aims to limit the heterogeneity and controversy in defining DCI for research and, potentially, clinical application, combining both imaging and clinical assessments in the determination of DCI. The future direction for validation of this proposed reference standard through prospective studies may help to move forward both clinical care and research in this field.

Disclosures: Pina Sanelli-RELATED: Grant: National Institute of Neurological Disorders and Stroke, a component of the National Institutes of Health, grant 5K23NS058387. Ajay Gupta—UNRELATED: Grants/Grants Pending: Association of University Radiologists GE Radiology Research and Academic Fellowship.* *Money paid to the institution.

\section{REFERENCES}

1. Hop JW, Rinkel GJ, Algra A, et al. Case-fatality rates and functional outcome after subarachnoid hemorrhage: a systematic review. Stroke 1997;28:660-64

2. Stegmayr B, Eriksson M, Asplund K. Declining mortality from subarachnoid hemorrhage: changes in incidence and case fatality from 1985 through 2000. Stroke 2004;35:2059-63

3. Diringer MN, Bleck TP, Claude Hemphill J 3rd, et al. Critical care management of patients following aneurysmal subarachnoid hemorrhage: recommendations from the Neurocritical Care Society's Multidisciplinary Consensus Conference. Neurocrit Care 2011;15:211-40

4. Roos YB, de Haan RJ, Beenen LF, et al. Complications and outcome in patients with aneurysmal subarachnoid haemorrhage: a prospective hospital based cohort study in the Netherlands. J Neurol Neurosurg Psychiatry 2000;68:337-41

5. Hijdra A, Van Gijn J, Stefanko S, et al. Delayed cerebral ischemia after aneurysmal subarachnoid hemorrhage: clinicoanatomic correlations. Neurology 1986;36:329-33

6. Vergouwen MD, and Participants in the International Multi-Disciplinary Consensus Conference on the Critical Care Management of Subarachnoid Hemorrhage. Vasospasm versus delayed cerebral ischemia as an outcome event in clinical trials and observational studies. Neurocrit Care 2011;15:308-11

7. van der Schaaf IC, Ruigrok YM, Rinkel GJ, et al. Study design and outcome measures in studies on aneurysmal subarachnoid hemorrhage. Stroke 2002;33:2043-46

8. Reichman M, Gold R, Greenberg E, et al. Validation of a new reference standard for the diagnosis of vasospasm. Acad Radiol 2010;17:1083-89
9. Sanelli PC, Anumula N, Gold R, et al. Outcomes-based assessment of a new reference standard for delayed cerebral ischemia related to vasospasm in aneurysmal subarachnoid hemorrhage. Acad Radiol 2012;19:1066-74

10. Vergouwen MD, Vermeulen M, van Gijn J, et al. Definition of delayed cerebral ischemia after aneurysmal subarachnoid hemorrhage as an outcome event in clinical trials and observational studies: proposal of a multidisciplinary research group. Stroke 2010;41:2391-95

11. Vergouwen MD, Ilodigwe D, Macdonald RL. Cerebral infarction after subarachnoid hemorrhage contributes to poor outcome by vasospasm-dependent and -independent effects. Stroke 2011;42: 924-29

12. Dankbaar JW, Rijsdijk M, van der Schaaf IC, et al. Relationship between vasospasm, cerebral perfusion, and delayed cerebral ischemia after aneurysmal subarachnoid hemorrhage. Neuroradiology 2009;51:813-19

13. Etminan N, Vergouwen MD, Ilodigwe D, et al. Effect of pharmaceutical treatment on vasospasm, delayed cerebral ischemia, and clinical outcome in patients with aneurysmal subarachnoid hemorrhage: a systematic review and meta-analysis. J Cereb Blood Flow Metab 2011;31:1443-51

14. Centre For Evidence Based Medicine. OCEBM Levels of Evidence Working Group. Oxford levels of evidence 1. http://www.cebm.net/ index.aspx? $=5653$. Accessed March 29, 2013

15. Kreiter KT, Mayer SA, Howard G, et al. Sample size estimates for clinical trials of vasospasm in subarachnoid hemorrhage. Stroke 2009;40:2362-67

16. Frontera JA, Fernandez A, Schmidt JM, et al. Defining vasospasm after subarachnoid hemorrhage: what is the most clinically relevant definition? Stroke 2009;40:1963-68

17. Vergouwen MD, Etminan N, Ilodigwe D, et al. Lower incidence of cerebral infarction correlates with improved functional outcome after aneurysmal subarachnoid hemorrhage. J Cereb Blood Flow Metab 2011;31:1545-53

18. Pickard JD, Murray GD, Illingworth R, et al. Effect of oral nimodipine on cerebral infarction and outcome after subarachnoid haemorrhage: British aneurysm nimodipine trial. $B M J$ 1989;298:636-42

19. Etminan N, Vergouwen MD, Macdonald RL. Angiographic vasospasm versus cerebral infarction as outcome measures after aneurysmal subarachnoid hemorrhage. Acta Neurochir Suppl 2013;115:33-40

20. Fisher CM, Roberson GH, Ojemann RG. Cerebral vasospasm with ruptured saccular aneurysm-the clinical manifestations. Neurosurgery 1977;1:245-48

21. Rabinstein AA, Friedman JA, Weigand SD, et al. Predictors of cerebral infarction in aneurysmal subarachnoid hemorrhage. Stroke 2004;35:1862-66

22. Fergusen S, Macdonald RL. Predictors of cerebral infarction in patients with aneurysmal subarachnoid hemorrhage. Neurosurgery 2007;60:658-67, discussion 667

23. Crowley RW, Medel R, Dumont AS, et al. Angiographic vasospasm is strongly correlated with cerebral infarction after subarachnoid hemorrhage. Stroke 2011;42:919-23

24. Wintermark M, Ko NU, Smith WS, et al. Vasospasm after subarachnoid hemorrhage: utility of perfusion CT and CT angiography on diagnosis and management. AJNR Am J Neuroradiol 2006;27:26-34

25. Washington CW, Zipfel GJ, and Participants in the International Multi-Disciplinary Consensus Conference on the Critical Care Management of Subarachnoid Hemorrhage. Detection and monitoring of vasospasm and delayed cerebral ischemia: a review and assessment of the literature. Neurocrit Care 2011;15:312-17

26. Schmidt JM, Wartenberg KE, Fernandez A, et al. Frequency and clinical impact of asymptomatic cerebral infarction due to vasospasm after subarachnoid hemorrhage. J Neurosurg 2008;109:1052-59

27. Unterberg AW, Sakowitz OW, Sarrafzadeh AS, et al. Role of bedside 
microdialysis in the diagnosis of cerebral vasospasm following aneurysmal subarachnoid hemorrhage. J Neurosurg 2001;94:740-49

28. Wagner M, Steinbeis P, Guresir E, et al. Beyond delayed cerebral vasospasm: infarct patterns in patients with subarachnoid hemorrhage. Clin Neuroradiol 2013;23:87-95

29. Sanelli PC, Anumula N, Johnson CE, et al. Evaluating CT perfusion using outcome measures of delayed cerebral ischemia in aneurysmal subarachnoid hemorrhage. AJNR Am J Neuroradiol 2013; 34:292-98

30. Dankbaar JW, de Rooij NK, Velthuis BK, et al. Diagnosing delayed cerebral ischemia with different $C T$ modalities in patients with subarachnoid hemorrhage with clinical deterioration. Stroke 2009;40: 3493-98

31. Greenberg ED, Gold R, Reichman M, et al. Diagnostic accuracy of CT angiography and CT perfusion for cerebral vasospasm: a metaanalysis. AJNR Am J Neuroradiol 2010;31:1853-60

32. Wintermark M, Reichhart M, Cuisenaire $\mathrm{O}$, et al. Comparison of admission perfusion computed tomography and qualitative diffusion-and perfusion-weighted magnetic resonance imaging in acute stroke patients. Stroke 2002;33:2025-31

33. Schramm P, Schellinger PD, Klotz E, et al. Comparison of perfusion computed tomography and computed tomography angiography source images with perfusion-weighted imaging and diffusionweighted imaging in patients with acute stroke of less than 6 hours' duration. Stroke 2004;35:1652-58

34. Chieregato A, Tanfani A, Noto A, et al. Cerebral blood flow thresholds predicting new hypoattenuation areas due to macrovascular ischemia during the acute phase of severe and complicated aneurys- mal subarachnoid hemorrhage: a preliminary study. Acta Neurochir Suppl 2008;102:311-16

35. Peerdeman SM, van Tulder MW, Vandertop WP. Cerebral microdialysis as a monitoring method in subarachnoid hemorrhage patients, and correlation with clinical events-a systematic review. J Neurol 2003;250:797-805

36. Sarrafzadeh A, Haux D, Sakowitz O, et al. Acute focal neurological deficits in aneurysmal subarachnoid hemorrhage: relation of clinical course, CT findings, and metabolite abnormalities monitored with bedside microdialysis. Stroke 2003;34:1382-88

37. Cerejo A, Silva PA, Vilarinho A, et al. Intraoperative brain oxygenation monitoring and vasospasm in aneurysmal subarachnoid hemorrhage. Neurol Res 2012;34:181-86

38. Yokose N, Sakatani K, Murata Y, et al. Bedside assessment of cerebral vasospasms after subarachnoid hemorrhage by near infrared time-resolved spectroscopy. Adv Exp Med Biol 2010;662:505-11

39. Yokose N, Sakatani K, Murata Y, et al. Bedside monitoring of cerebral blood oxygenation and hemodynamics after aneurysmal subarachnoid hemorrhage by quantitative time-resolved near-infrared spectroscopy. World Neurosurg 2010;73:508-13

40. Anderson GB, Ashforth R, Steinke DE, et al. CT angiography for the detection of cerebral vasospasm in patients with acute subarachnoid hemorrhage. AJNR Am J Neuroradiol 2000;21:1011-15

41. Wintermark M, Dillon WP, Smith WS, et al. Visual grading system for vasospasm based on perfusion CT imaging: comparisons with conventional angiography and quantitative perfusion CT. Cerebrovasc Dis 2008;26:163-70 